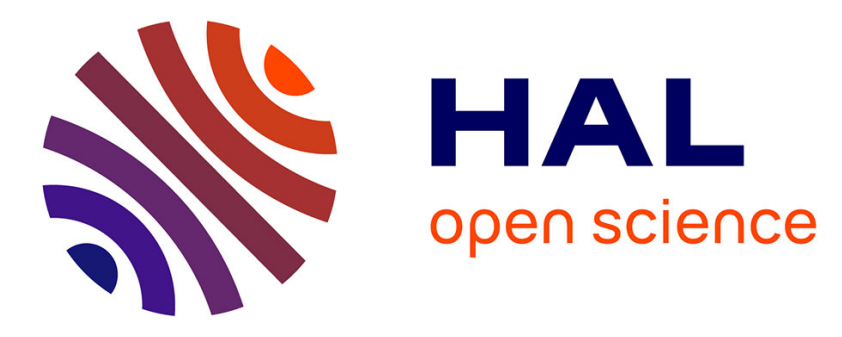

\title{
Application of multi-perspective modeling and holistic simulation to Urban Transportation Systems
}

Mamadou Kaba Traoré, Oumar Maiga, Mamadou Traoré, Youssouf Koné, O. Maïga, K.M. Traoré

\section{- To cite this version:}

Mamadou Kaba Traoré, Oumar Maiga, Mamadou Traoré, Youssouf Koné, O. Maïga, et al.. Application of multi-perspective modeling and holistic simulation to Urban Transportation Systems. The 32nd European Modeling \& Simulation Symposium, Sep 2020, Athènes, Greece. pp.93-102, 10.46354/i3m.2020.emss.013 . hal-02977165

\section{HAL Id: hal-02977165 \\ https://hal.science/hal-02977165}

Submitted on 24 Oct 2020

HAL is a multi-disciplinary open access archive for the deposit and dissemination of scientific research documents, whether they are published or not. The documents may come from teaching and research institutions in France or abroad, or from public or private research centers.
L'archive ouverte pluridisciplinaire HAL, est destinée au dépôt et à la diffusion de documents scientifiques de niveau recherche, publiés ou non, émanant des établissements d'enseignement et de recherche français ou étrangers, des laboratoires publics ou privés. 
$32^{\text {nd }}$ European Modeling \& Simulation Symposium

$17^{\text {th }}$ International Multidisciplinary Modeling \& Simulation Multiconference

\title{
Application of Multi-Perspective Modeling and Holistic Simulation to Urban Transportation Systems
}

\author{
Youssouf Koné1,*, Oumar Maïga² and Mamadou K. Traoré3 \\ ${ }^{1}$ LIMOS CNRS UMR 6158, Université Clermont Auvergne 49 bd François Mitterrand CS 60032, Clermont-Ferrand, \\ 63001, France \\ ${ }^{2}$ Université des Sciences, des Techniques et des Technologies de Bamako, Hamdalaye ACI 2000, Rue 405, Porte \\ 359, Bamako, Mali \\ 3IMS CNRS UMR 5218, Université de Bordeaux 351 cours de la Libération CS 10004, Bordeaux, 33405, France \\ ${ }^{*}$ Corresponding author. Email address: youssouf.kone@etu.uca.fr
}

\begin{abstract}
As Urban Transportation Systems (UTS) are becoming central in our daily lives, they are also gaining in complexity due to their ever-increasing interdependence with other socio-economic dimensions (such as climate, demography and environmental policies, to name a few). This paper presents a multi-perspective modeling and holistic simulation framework that can address such a complexity. It is based on the holistic integration of models that have independently been built from the respective perspectives of various but interdependent dimensions. The resulting model can answer questions that any of the perspectivespecific models cannot in isolation.
\end{abstract}

Keywords: Urban Transportation System (UTS); Modeling and Simulation (M\&S); Multi-Perspective Modeling and Holistic Simulation (MPM\&HS); High Level Language for System Specification (HiLLS)

\section{Introduction}

Transportation systems play an important role in social and economic activities in modern countries. The increase of traffic flow in cities often leads to traffic jams, pollution and accidents. An Urban Transportation System (UTS) is a complex system consisting of a set of autonomous constituents interacting with each other. It is a system of systems, due to the fact that each constituent of the transport system represents a more or less complex system. The study of such a complex system linking several other systems is a delicate task that deserves special consideration and attention.

Over the years, Modeling and Simulation (M\&S) has been used as the main technique to study and forecast the behavior of urban traffic for management, planning and security purposes. Traditionally, traffic flow models have been classified as macroscopic, microscopic and mesoscopic (Hoogendoorn and Bovy, 2001). Macroscopic models are at the basis of urban traffic flow modeling and analysis. In macroscopic models, traffic flows are defined by aggregated variables of the traffic: flow, density and average speed. While macroscopic models can be used to represent behavior of traffic flow in large-scale areas, it cannot be used to determine causes of traffic jams and detailed information at the level of vehicles. In the microscopic models, the individual characteristics of the vehicles are represented. Behaviors and interactions with the environment are studied individually for each vehicle. Mesoscopic models are

(C) 2020 The Authors. This article is an open access article distributed under the terms and conditions of the Creative Commons Attribution (CC BY-NC-ND) license (https://creativecommons.org/licenses/by-nc-nd/4.0/). 
intermediate representation of traffic flow between macroscopic and microscopic approaches, where the focus is made on platoons of vehicles rather than on individual vehicles or the aggregated population of all vehicles (this allows e.g. analyzing traffic jam, based on the behavior of platoons of vehicle, each behavior being an average profile of the vehicles that constitute the platoon).

In this paper, we present a multi-perspective modeling framework and holistic simulation of urban transportation systems allowing to:

- offer a unifying approach to conventional models making available to the expert in the field of urban transportation systems a range of traffic theory models;

- build an ontology for the modeling and simulation of a structured transportation system at four levels: system, facet, scale and model levels;

- integrate different perspectives of the urban transportation system into a global model for holistic simulation of the system.

The remaining of this paper is organized as follows. Section 2 presents related works and underlines the originality of our approach. Section 3 gives a brief presentation of the multi-perspective modeling and holistic simulation approach. Section 4 shows how the approach has been customized for Urban Transportation Systems (UTS) and presents an application of the resulting framework, using realworld UTS. Section 5 concludes the paper.

\section{Related works}

Depending on the level of detail desired, the traffic theory defines four major approaches to modeling transportation systems: the macroscopic, mesoscopic, microscopic, and combined approaches.

The macroscopic UTS simulation approach mathematically models the UTS as one dimensional fluid characterized by its density, flow rate and speed (Gazis, 2006). This approach makes use of two categories of equations: first order equations (Lighthill et al., 1955a; Lighthill et al., 1955b), (Richards, 1956) and second order equations (Payne, 1971). Simulators implementing this approach are most useful for the simulation of wide-area road networks where details are not important; they give only global information about traffic flow and neglect details related to vehicles, drivers driving styles and the impact of interactions between vehicles. A limitation of this approach is that it does not allow representing the propagation of the congestion on a network, and travel times are not very representative (Abouaïssa et al., 2015). Examples of tools and frameworks implementing the macroscopic approach to traffic modeling and simulation are: AIMSUN 2 (Barcelo et al., 1998), METNET (Kotsialos et al., 2002), etc.
The microscopic approach is most used in UTS simulation because of the required details to understand the impact of individual vehicles and their interactions on the congestions that appear, and to evaluate different control, operations and management strategies. At this level, every vehicle is characterized by its spatial position, its speed, and its interactions with neighboring vehicles. Microscopic models include car-following models (Chandler et al. 1958), (Gazis et al., 1961), (Gerlough and Huber, 1975), (Gazis et al., 1959), (Bando et al., 1995a; Bando et al., 1995b), (Gipps, 1981), lane changing models (Gipps, 1986), and gap acceptance algorithms (Daganzo, 1981). The advantages of this approach include the exact representation of the geometry of the road network and the representation of the individual behavior of the vehicles that makes it possible to know the source of congestion (Abouaïssa et al., 2015). A limitation of this approach is that it is not easily usable for the wide-area road networks and uses high computing times (Dimon, 2012). Examples of tools and frameworks implementing the microscopic approach to traffic modeling and simulation are: AIMSUN (Barcelo et al., 1998), VISSIM (Barcelo et al., 2010), SUMO (Krajzewicz et al., 2002), CORSIM (Owen et al., 2000), Simtraffic (Sorenson and Collins, 2000), PARAMICS (Cameron and Duncan, 1996), etc.

Mesoscopic simulators combine the levels of details of macroscopic and microscopic simulators. Vehicles are grouped in pelotons which are considered as single entities having their own position and speed. The Advantages are the simplification of the models of behavior and the fact that this approach allows to approach problems of congestion in an urban environment on a large scale (Dimon, 2012). Examples of tools and frameworks implementing the mesoscopic approach to traffic modeling and simulation are: CONTRAM (Leonard et al., 1989), AIMSUN 2 (Barcelo et al., 1998), DYNAMEQ (Mahut, 2001), etc.

While macroscopic, mesoscopic, and microscopic approaches focus on the scale at which the problem is studied, they do not take into account the influence of changes in external phenomena. For example, drivers' behavior can be influenced by the change visibility due to the change of weather, which itself can be impacted by the pollution generated by vehicles. Also, the density and distribution of users flow in UTS is impacted by population dynamics and the urban development, while it contributes to that development too. The multi-perspective modeling and holistic simulation approach has been proposed to address this concern. This paper shows how it can be customized to UTS.

\section{Multi-Perspective Modeling and Holistic Simulation}

Multi-Perspective Modeling and Holistic Simulation (MPM\&HS) is a recently introduced simulation approach (Djitog et al., 2017), (Traoré et al., 2018) and 
(Traoré, 2019), which relies on the principle that a complex system requires various levels of understanding (which we call perspectives or facets) be independently provided and later combined holistically, as every perspective influences every other one. A perspective (or facet) is an objectivedriven focus done on a given system. As an example, multiple perspectives are considered in the area of healthcare systems modeling and simulation, ranging from disease spreading, through population dynamics, to health resources allocation. Each perspective is the topic of numerous studies where knowledge and results are established in the shape of conventional models.

The concept of perspective can be well-captured by the notion of experimental frame (EF) as introduced in (Zeigler, 1976) and more developed in (Zeigler, 1984), with a formalization suggested in (Traoré and Muzy, 2006). The EF is the set of conditions under which the system is being observed, and is operationally formalized to capture the objectives of the study. It drives the elaboration of the system's model as a set of rules or mathematical equations that give an abstract representation of the system, which is used to replicate the behavior of that system. Therefore, the objective-driven model that is built is necessarily perspective-specific. Notice that the specification of such model can provide multiple views, i.e., various aspects of the same system seen from a given perspective (like static, dynamic and functional views of a healthcare system taken from the perspective of disease spreading). Such a specification can also be composed of multiple formalisms (such as system dynamics, Petri net, cellular automata, etc.).

MPM\&HS has two parts: (i) multi-perspective modeling MPM), and (ii) holistic simulation (HS).

\subsection{Multi-Perspective Modeling (MPM)}

The first part of MPM\&HS, namely Multi-Perspective Modeling (MPM), relies on the idea that different levels of explanation can be obtained from a given system by repeatedly studying the system under various perspectives, each of which expressed as an $\mathrm{EF}$, as depicted by Figure 1. In other words, the same system is subject to various objective-driven studies (squares around the complex system, in Figure 1), leading to many perspective-specific models (facet model in Figure 1) and related EFs (specific EF in Figure 1). These perspective-specific EFs are elaborated to provide answers to questions of very different nature about the same system. Consequently, each of them is coupled with its corresponding model to derive results of interest in this perspective. Noteworthy are parameters of each perspectivespecific model, as a way to approximate abstractions from other perspectives. Indeed, each perspectivespecific model abstracts the influences that are due to other perspectives by means of parameters which values explicitly reflect implicit assumptions and simplifications done about these influences.

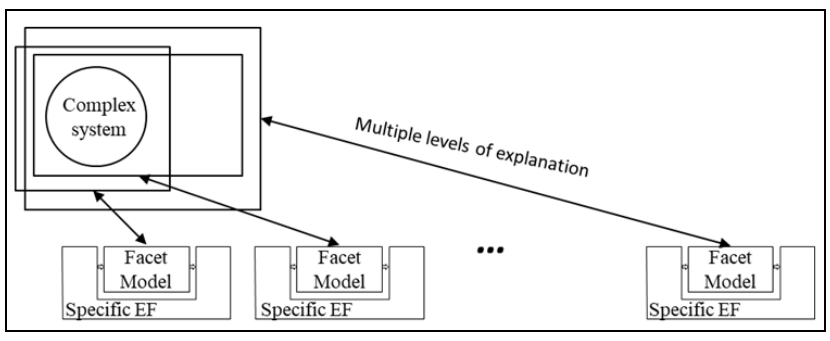

Figure 1. Multi-Perspective Modeling approach

MPM cannot be efficiently realized without a disciplined approach. Indeed, the identification of all possible perspectives, or at least the ones of major interest, is not obvious. (Zeigler et al., 2018) recently formulated fundamental requirements for M\&S of modern complex systems as follows: "develop an organizational ontology that supports combinatorial model compositions, with major facets at the top level to ensure macro behavior and their refinement into meso and micro behaviors, and a large spectrum of models at the bottom level for combinatorial composition". We present here a disciplined approach to building ontology for the M\&S of a domain of interest, which meets these requirements. It is essential that the ontology to be built provides, at some general level, a formal way to capture all the knowledge that might be in the range of M\&S of the domain for which it is likely to be used. Therefore, it must capitalize on the abstractions used for the simulation of the entire targeted domain, beyond perspective-specific modeling. Thus, we suggest a domain-independent M\&S ontology that adopts a layered analysis approach, which is captured in Figure 2 using the System Entity Structure (SES) ontological framework (Zeigler, 1984).

SES is a declarative knowledge representation scheme that characterizes the structure of a family of models in terms of decompositions, component taxonomies, and coupling specifications and constraints. Models are represented by boxes. They can have variables, which can be assigned a value within a given range. An Aspect expresses a way of decomposing a model into more detailed parts. A Multi-Aspect is an aspect for which the component models are all of one kind. A Specialization is a category or family of specific forms that a model can assume. SES axioms are (Zeigler and Sarjoughian, 2017): uniformity, strict hierarchy, alternating mode, valid brothers, attached variables, and inheritance. Uniformity forces that any two nodes with the same labels have isomorphic subtrees. Strict hierarchy prohibits a label from appearing more than once down any path of the tree. Alternating mode states that, if a node is a model, then the successor is either Aspect or Specialization, and vice versa. Valid brothers forbid having two brothers with the same label. Attached variables constrains that variable types attached to the same model shall have distinct names. Inheritance asserts that Specialization inherits all variables and Aspects from the parent model to the children models. (Zeigler and Hammonds, 2007) provide a formal set- 
theoretic characterization of the SES that shows how the axioms are satisfied.

The domain-independent ontology is meant to be instantiated in the analysis of any new domain of interest in view of its M\&S. Such an instantiation provides the domain-specific ontology that will drive the MPM\&HS process of the targeted domain. As depicted by Figure 2, the following layers are defined:

- Facet level is where specializations of the class of systems that characterizes the domain of interest are highlighted, and cumulative aspects of a domain system are separated.

- Scale level is where major spatial and temporal scales are emphasized. The microscopic level is the finest representation of a system entity that takes into account the individual behavior of each entity and their interactions with the environment. The macroscopic level is the most comprehensive representation of the system, which is considered as a whole. The mesoscopic level is an intermediate representation between the microscopic and macroscopic levels that considers the behaviors of groups of entities with common characteristics.

- Model level is where legacy models often originating from decades of theoretical findings are identified as reusable artefacts to be selected and integrated in new studies. These different models are specified and implemented in a library of ready-to-use simulation models.

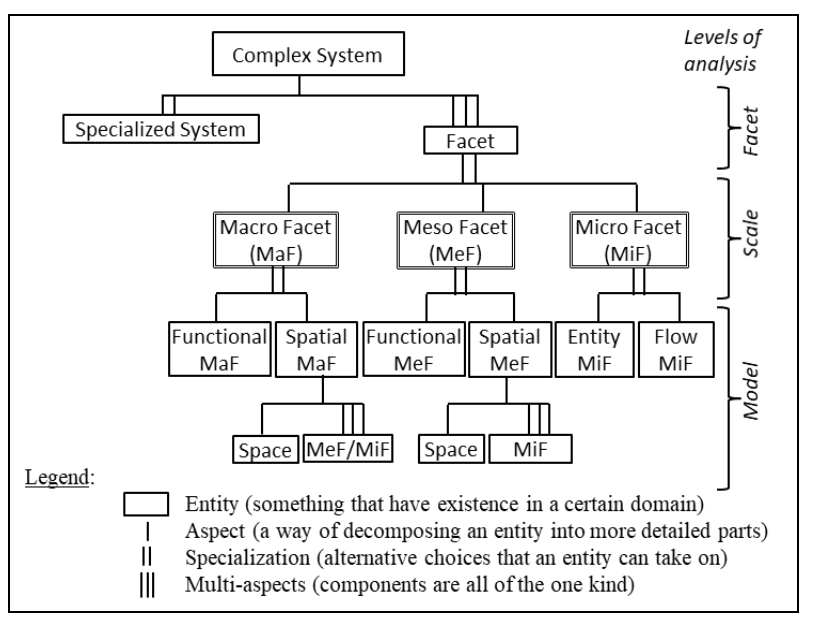

Figure 2. Domain-independent Ontology for Complex Systems M\&S (O4CS)

As such, the domain-independent ontology (O4CS) suggests a generic organization, where the family of alternative models displayed at the leaves of $\mathrm{O} 4 \mathrm{CS}$ has to be implemented and saved in a model base (called MB4CS and represents by figure 3). Once the models are saved, they can be retrieved from their repository and reused to design complex systems. Legacy and conventional models (which we call white models) are models available in the literature, which are based on established theories, generic for a class of systems, and parameterized to be reusable in various similar situations. Examples of such models are the ones mentioned in the previous sub-section. Specific models (which we call gray models) are the ones implemented in the library by the user, either by adapting a white model to a specific system, or by building from scratch for her/his specific purposes.

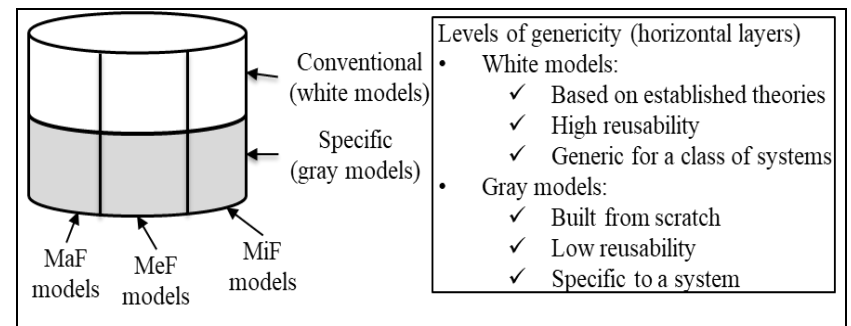

Figure 3. Domain-independent model base for complex systems simulation (MB4CS)

\subsection{Holistic Simulation}

While in practice, perspective-specific models are executed in isolation, i.e., without recourse to the processes from other perspectives, all these models are related in reality, since they depict various abstractions of the same system. However, building a monolithic highly detailed mega-model that involves all inter-influencing factors is not viable. Therefore, the second part of MPM\&HS, namely Holistic Simulation (HS), suggests gluing the perspectives together by enabling live exchanges of information between models from different perspectives through integrators, as shown in Figure 4. That way, a holistic view is obtained, which encompasses isolated perspective-specific simulations and their mutual influences, without a drastic increase of complexity. Such integration is done by dynamically feeding the parameters of a focused model in a given perspective with the outputs of models from other perspectives. When the perspective-specific models are holistically integrated that way, a holistic EF can be elaborated and questions that are transversal to different perspectives can then be addressed. This holistic EF is to be coupled with the resulting holistic model to derive results that cannot be accurately addressed in any of the perspective taken alone. Technically, this is realized by creating a coordinating model, which translates output received from models into new values for the parameters of other models. (Zeigler et al., 2019) gave a formal specification of such integration in the context of DEVS M\&S (Zeigler 1976; Zeigler et al., 2000). 


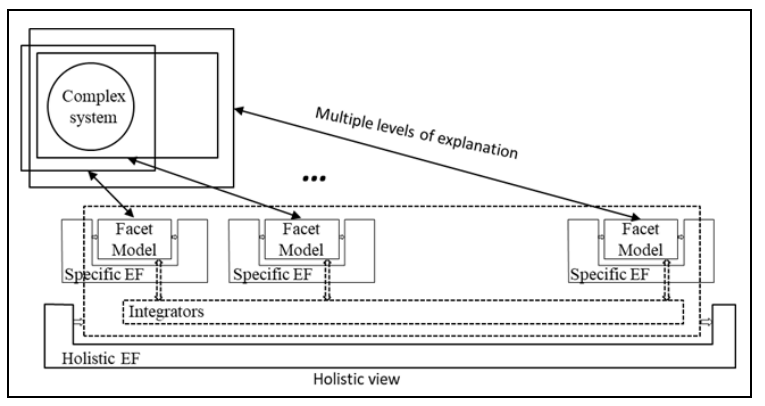

Figure 4. General principle of Holistic Simulation

MPM\&HS is fundamentally a hybrid simulation framework as well as a multi-paradigm framework, as models of the ontology can be continuous or discrete, with multiple levels of abstraction that can expressed in various formalisms. To allow a unified specification, a pivotal language named HiLLS (for High Level Systems Specification) is proposed (Aliyu et al., 2016). It is a visual language which operational semantics for simulation is given in DEVS (while other semantics are given in other languages for other kinds of analysis). HiLLS combines the expressive power of automata theory (through which DEVS concepts are visually captured), object oriented methodology (so that encapsulation, composition and inheritance are supported), and logic (which allows variables, parameters and operations be captured as predicates, so that they can easily be manipulated symbolically).

\section{Application to UTS}

The whole MPM\&HS methodological process is driven by the SES-based domain-independent ontology O4CS, as depicted by Figure 5 . The analysis of a specific domain is guided by O4CS (and MB4OCS) and results in a domain-specific ontology and its corresponding model base. Then, within that domain, the modeler develops multi-perspective models to meet intended objectives of a given study, through the pruning of the domain-specific ontology and the selection of appropriate components in the domainspecific model base. Pruning is the process of extracting from the domain-specific ontology a specific system configuration, selecting particular subsets of Aspects, particular cardinalities of MultiAspects, and particular instances of Specializations, and assigning values to the variables. Pruning of the ontology results in a multi-perspective model, where each top facet model is a multi-scale coupled model that addresses intra scale transfer issues. Facet models are then integrated in a holistic model through welldefined integrators that address inter-perspective scale transfer issues. The holistic model can then be simulated under various scenarios to answer questions of interest.

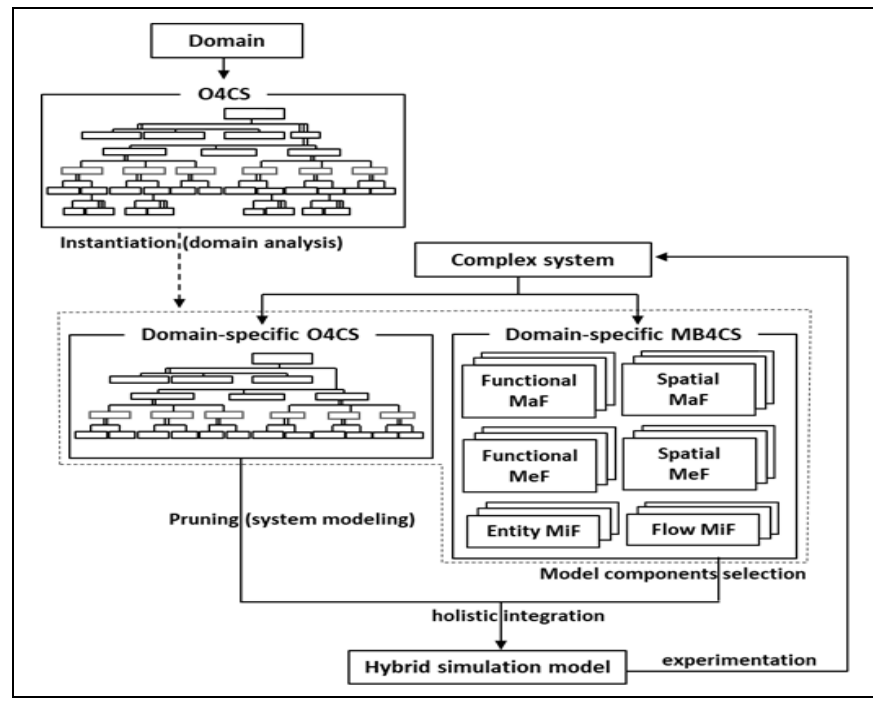

Figure 5. MPM\&HS methodological process

\subsection{UTS-specific ontology}

We propose to apply the MPM\&HS process to the domain of UTS. The resulting UTS-specific ontology is shown by Figure 6.brief statement of what is being reported in the article.

The system level is the highest level of knowledge representation in the domain of urban transportation systems and corresponds to the different categories of urban transportation systems. Each category can be studied as a juxtaposition of several perspectives. We distinguish several categories of transportation systems: pedestrian traffic, two-wheel traffic, automobile traffic, public transportation traffic. These different systems constitute transportation modes in urban transportation systems.

The facet level presents the different levels of abstraction of the system. The MPM\&HS approach recommended three perspectives: consumption, production and coordination. In the context of urban transport systems, the consumption perspective is the use of transportation infrastructure by users (population) to meet their travel needs. Production perspective corresponds to the put in place of transportation infrastructures (roads, intersections, parking, traffic light, toll roads, etc.) and police services for traffic regulation. Coordination perspective allows the combination of the other two perspectives (consumption and production).

The Scale level allows the spatio-temporal division of each perspective. Each of the three perspectives (consumption, production and coordination) can be observed according to the desired level of detail at different scales. In accordance with the MPM\&HS approach, we mainly distinguish three scales of representation: microscopic scale, macroscopic scale and mesoscopic scale. 


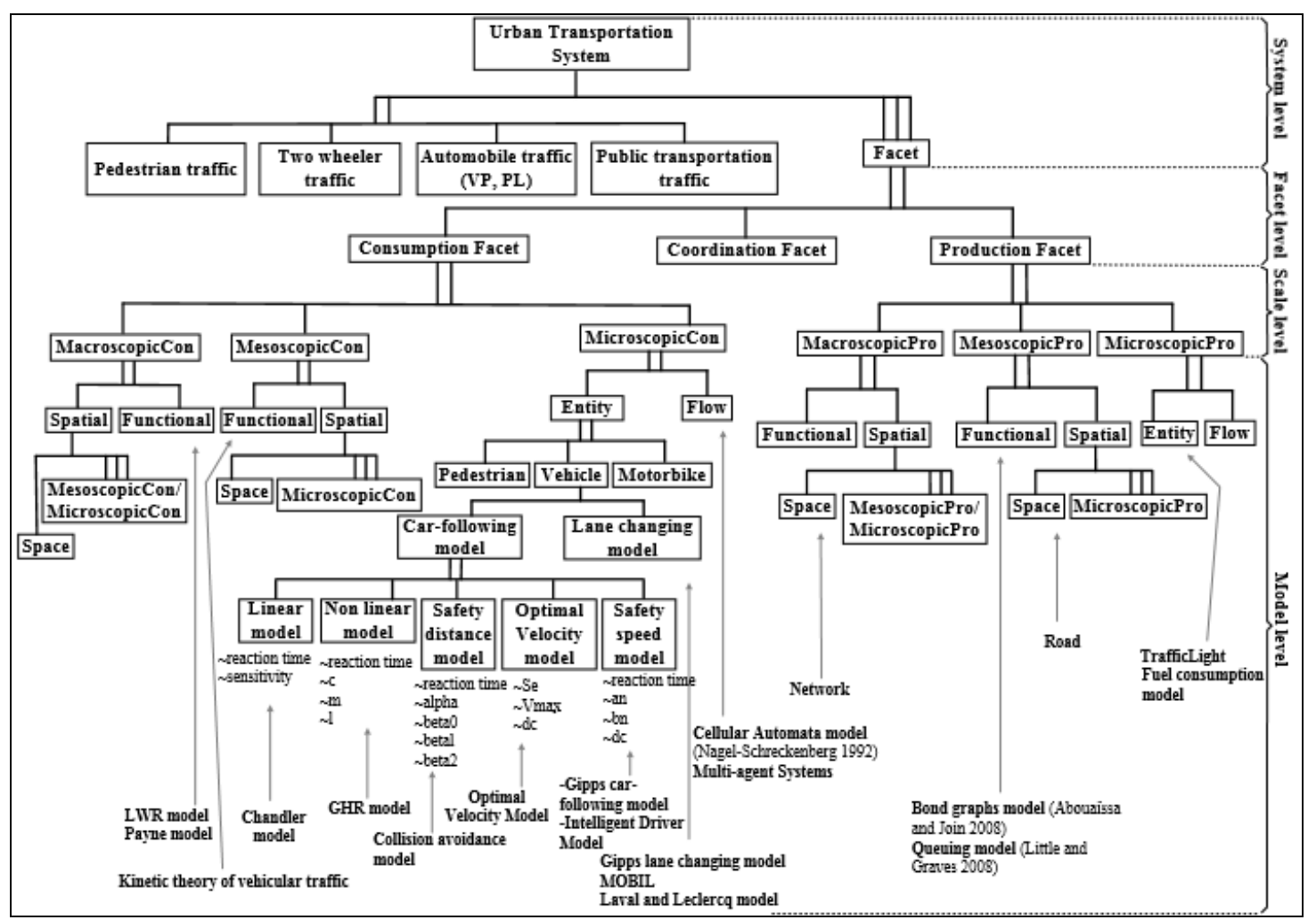

Figure 6. Ontology for urban transportation system M\&S

The model level gives the last level of the ontology which takes into account the different conventional models existing in the theory of the traffic. The conventional models are distributed among the different spatio-temporal scales of the perspectives. On a macroscopic scale, models can be functional models expressed using mathematical equations or spatial models that describe the space containing microscopic or mesoscopic entities. At the mesoscopic scale, the models can also be functional or spatial models. At the microscopic scale, models can be feature models that describe the individual characteristics and behaviors of entities or flow patterns that represent the behavioral patterns that an individual in the system may experience. There are several types of entities: pedestrian, motorcyclist, vehicle, etc. A vehicle is generally characterized by a car-following model (longitudinal movement) and a lane change model (lateral movement).

Several conventional models have been proposed in the literature. In the macroscopic domain, we have: LWR model (Lighthill and Witham, 1995; Richards, 1996), (Payne, 1971) model, etc. In microscopic domain, we have following models, lane change models and cellular automaton models. Main carfollowing models are: linear model of (Chandler et al., 1958), GHR model of (Gazis et al., 1959), model of (Gipps, 1981), model OVM (Bando et al., 1995), IDM model (Treiber et al., 2000), etc. Lane change models are: the model of (Gipps, 1986), the MOBIL model
(Kesting et al., 2007), etc. Models of cellular automaton are: the model of (Nagel and Schreckenberg, 1992), the model of (Brockfeld et al., 2001), etc. In mesoscopic domain, we have: the model of (Prigogine and Herman, 1971).

\subsection{UTS-specific Model Base}

We have specified and implemented conventional models in the model base for urban systems (MB4UTS). Each conventional model is specified in HiLLS formalism. The components library (Model Base) is made up of the various conventional models implemented.

Figure 7 illustrates the HiLLS specification of Chandler's model (Chandler et al., 1958). It is described by a box similar to a UML class with an additional horizontal compartment and two vertical compartments. The left (respectively right) hand side vertical compartment has input (respectively output) ports attached to it. The concept of port is defined as in DEVS (Zeigler, 1976). All declarations (whether ports or any other variables or functions) are done in firstorder logic, using the $\mathrm{Z}$ declaration schema approach (Smith, 2002). The top horizontal compartment contains the name of the model, and the declaration of its parameters. The immediate compartment below contains the declaration of state variables. The third compartment from top contains the definitions of operation schemas that use and manipulate all variables, including parameters and ports. Therefore, 
while a message received on some given input port causes a change of the internal state of the model, a call to some given modifier operation causes a change of value of some given parameter. The bottom compartment contains the system's behavior described by the configuration transition diagram, a graph which vertices represent the configuration set and which edges represent configuration-toconfiguration transitions that can occur in the system. A configuration corresponds to an assignment of specific values or constraints to each of the state variables. A configuration depicts a set of properties that several states share.

Figure 7 displays 2 configurations. The "cognition" configuration is a 4-compartments box, which respectively contains: (i) the label of the configuration, (ii) the logic specification of its properties, such as the assignment of values and constraints to state variables, (iii) its duration, which corresponds in DEVS to the value of the time advance function, and (iv) the description of activities to be carried out when the system is in this configuration (void, in this case). The "driving" configuration is defined similarly, but is reduced to a 3-compartments box, since its duration is $+\infty$, which is signaled by a double line at the right hand edge of the box. Configuration transitions are denoted by different labeled arrows with the operations accompanying the transitions (for update of state variables, when needed) as part of the labels. For internal transition, the value sent on the output port (signaled by "!") is also part of the label. For external transition, the label includes the triggering condition on the receipt of a value on a port (signaled by "?") and the time elapsed by the model in its current configuration (if needed). A black circle allows making reference to the initial configuration of the model.

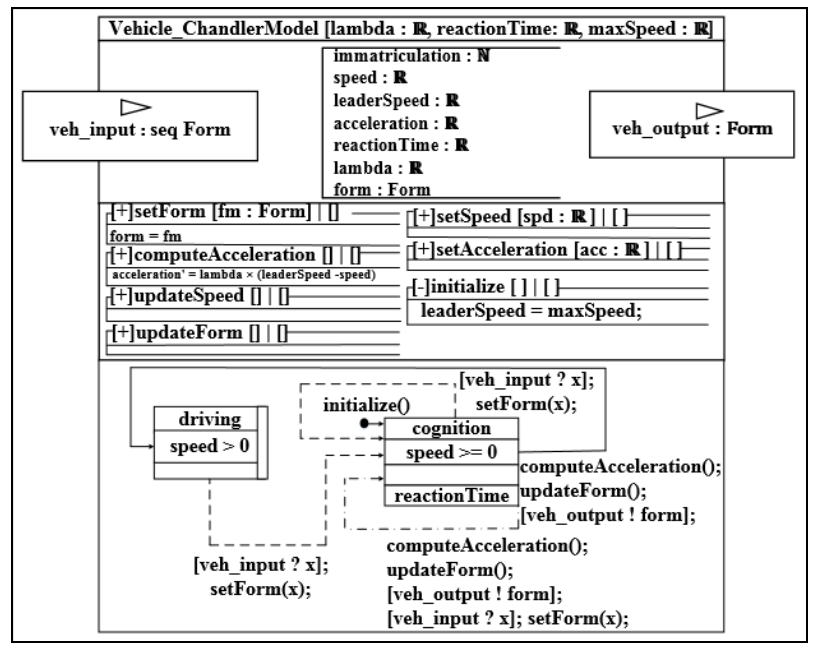

Figure 7. HiLLS-specified Chandler's model

\subsection{UTS model derivation}

Let's consider a road network consisting of two crossings (Figure 8), which we want to study from both macroscopic and microscopic perspectives, in order to explore the reciprocal impact of detailed driver behavior and global traffic network dynamics.

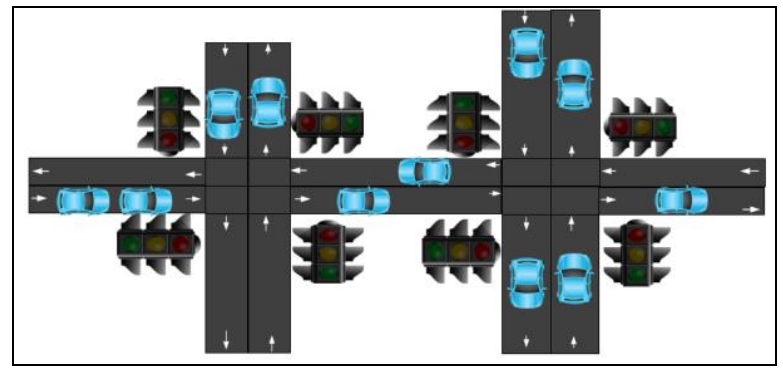

Figure 8. Road network

The structure of the model of such a system is obtained by pruning the UTS-specific ontology, in the way depicted by Figure 9, where the ontology has been used in two different perspectives. The first perspective, which corresponds to steps $1,2,7,8,9,10$ and 11 is obtained from the microscopic viewpoint of the traffic (i.e., individual driver behavior model). The second perspective, which corresponds to steps 1, 2, 3, 4,5 , and 6 is obtained from the macroscopic viewpoint of the traffic (i.e., the global traffic network dynamics).

Figure 10 shows how the resulting two models are, in one hand used in isolation in their respective experimental frames, and in the other hand integrated in a holistic simulation scheme, each model's parameters being updated with the outputs of the other model.

\section{Conclusion}

We have proposed a multi-perspective modeling and holistic simulation framework of Urban Transportation Systems (UTS), based on the generic MPM\&HS approach that offers a rigorous approach to M\&S of complex systems. Such a framework can help UTS experts and managers to easily derive multiple models from various perspectives and later connect them in a more inclusive view. The resulting model can answer questions that any of the perspectivespecific models cannot in isolation.

In the case study presented, the holistic model only integrates microscopic and macroscopic perspectives of UTS. Going farther, this model can be extended the same way to involve models from neighbor perspectives, such as pollution, weather, or population dynamics. This calls for the composition of domainspecific ontologies, which is part of our future work. Another critical aspect to be further considered is the validation of such a model. To that aim, we envision a two-step process, where individual models are first validated, and then an integration validation approach is considered for the holistic model. 


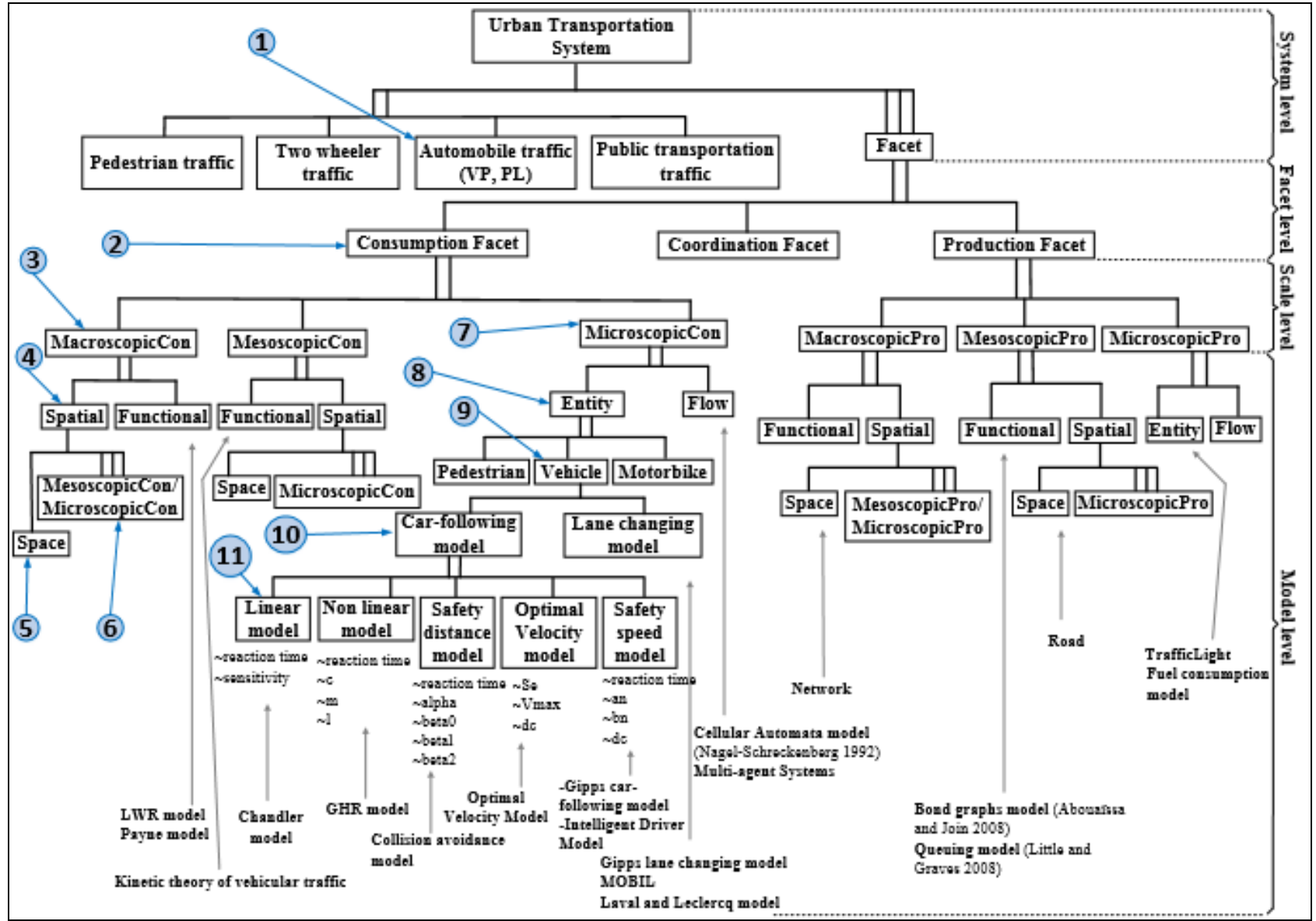

Figure 9. Pruning of the ontology to get the model of the case study

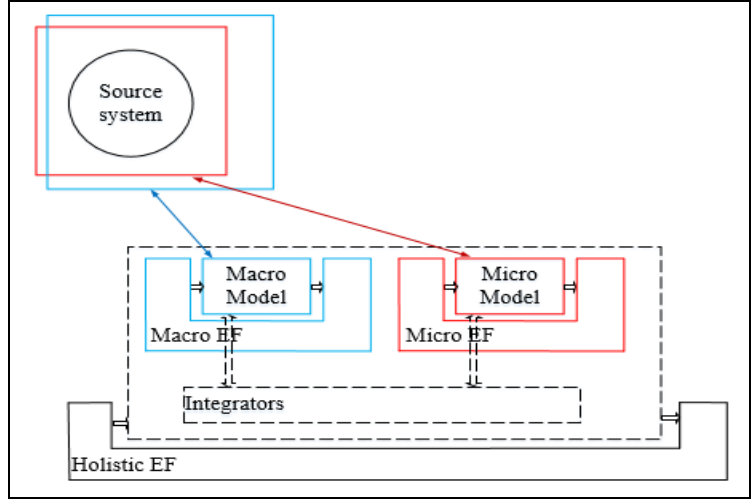

Figure 10. Holistic integration

\section{Acknowledgements}

This work was supported by the doctoral grant of the French Embassy in Mali, and the national "Training of Trainers" program of the republic of Mali.

\section{References}

Abouaïssa, H., Yoann, K., \& Morvan, G. (2015). The Modélisation hybride dynamique de flux de trafic. Thèse de Doctorat, Université d'Artois, France.
Aliyu, H. O., Maïga, O., \& Traoré, M. K. (2016). The high level language for system specification: A modeldriven approach to systems engineering International Journal of Modeling, Simulation, and Scientific Computing, 7(01), 1641003.

Bando, M., Hasebe, K., Nakayama, A., Shibata, A., \& Sugiyama, Y. (1995). Dynamical model of traffic congestion and numerical simulation. Physical review E, 51(2), 1035.

Bando, M., Hasebe, K., Nakanishi, K., Nakayama, A., Shibata, A., \& Sugiyama, Y. (1995). Phenomenological study of dynamical model of traffic flow. Journal de Physique I, 5(11), 1389-1399.

Barceló, J., Casas, J., Ferrer, J. L., \& García, D. (1998). Modelling advanced transport telematic applications with microscopic simulators: The case of aimsun. In Simulation technology, Science and Art, Proceedings of the 10th European Simulation Symposium, A. Bargiela and E. Kerckhoffs (Eds.) (pp. 362-367).

Barceló, J. (2010). Fundamentals of traffic simulation (Vol. 145, p. 439). New York: Springer.

Cameron, G. D., \& Duncan, G. I. (1996). PARAMICSParallel microscopic simulation of road traffic. The Journal of Supercomputing, 10(1), 25-53. 
Chandler, R.E., Herman, R., Montroll, E.W. (1958). Traffic dynamics: studies in car following, Operations Research, vol. 6, $\mathrm{n}^{\circ} 2$, pp. 165-184.

Daganzo, C. F. (1981). Estimation of gap acceptance parameters within and across the population from direct roadside observation. Transportation Research Part B: Methodological, 15(1), 1-15.

Dimon C. (2012). Contribution à la modélisation et la commande des réseaux de trafic routier, Thèse de Doctorat, Ecole Centrale de Lille, France.

Djitog, I., Aliyu, H. O., \& Traoré, M. K. (2017). MultiPerspective Modeling of Healthcare Systems. International Journal of Privacy and Health Information Management (IJPHIM), 5(2), 1-20.

Gazis, D.C., Herman, R., Potts, R.B. (1959). Carfollowing theory of steady state flow, Operations Research, vol. 7, n 4, pp. 499-505.

Gazis, D. C., Herman, R., \& Rothery, R. W. (1961). Nonlinear follow-the-leader models of traffic flow. Operations research, 9(4), 545-567.

Gazis, D. C. (2006). Traffic Theory. International Series in Operations Research \& Management Science.

Gerlough DL, Huber MJ (1975). Traffic flow theory: a monograph. TRB special report 165.

Gipps, P. G. (1981). A behavioural car-following model for computer simulation. Transportation Research Part B: Methodological, 15(2), 105-111.

Gipps, P. G. (1986). A model for the structure of lanechanging decisions. Transportation Research Part B: Methodological, 20(5), 403-414.

Hoogendoorn, S. P., \& Bovy, P. H. (2001). State-ofthe-art of vehicular traffic flow modelling. Proceedings of the Institution of Mechanical Engineers, Part I: Journal of Systems and Control Engineering, 215(4), 283-303.

Kotsialos A, Papageorgiou M, Diakaki C, Pavlis Y, Middelham F. (2002). Traffic flow modeling of large-scale motorway networks using the macroscopic modeling tool METANET. IEEE Trans Intell Transp Sys 3:282-292.

Krajzewicz D, Hertkorn G, Rössel C, Wagner P. (2002). SUMO (Simulation of Urban MObility) - an opensource traffic simulation. In Proceedings of the 4th middle East Symposium on Simulation and Modelling (MESM20002) (pp. 183-187).

Leonard, D. R., Gower, P., \& Taylor, N. B. (1989). CONTRAM: structure of the model (No. 78).

Lighthill, M. J., \& Whitham, G. B. (1955). On kinematic waves I. Flood movement in long rivers. Proc. R. Soc. Lond. A, 229(1178), 281-316.

Lighthill, M. J., \& Whitham, G. B. (1955). On kinematic waves II. A theory of traffic flow on long crowded roads. Proc. R. Soc. Lond. A, 229(1178), 317-345.
Mahut, M. (2001). A multi-lane extension of the Space-Time Queue Model of Traffic Dynamics. TRISTAN IV, Azores Islands.

Owen, L. E., Zhang, Y., Rao, L., \& McHale, G. (2000). Street and traffic simulation: traffic flow simulation using CORSIM. In Proceedings of the 32nd conference on Winter simulation (pp. 11431147). Society for Computer Simulation International.

Payne, H.J. (1971). Models for Freeway Traffic and Control. In: Bekey, G.A. (ed), Mathematical Models of Public Systems 1, 51-61.

Richards, P. I. (1956). Shock waves on the highway. Operations research, 4(1), 42-51.

Smith, G. (2012). The Object-Z specification language (Vol. 1). Springer Science \& Business Media.

Sorenson, D. K., \& Collins, J. (2000). Practical Applications of Traffic Simulation Using SimTraffic. In Compendium of Papers. Institute of Transportation Engineers 2000, District 6 Annual MeetingInstitute of Transportation Engineers.

Traoré, M. K., \& Muzy, A. (2006). Capturing The Dual Relationship Between Simulation Models and Their Context. Simulation Modelling Practice and Theory 14(2):126-142.

Traoré, M. K. (2019). Multi-Perspective Modeling and Holistic Simulation. Complexity Challenges in Cyber Physical Systems: Using Modeling and Simulation (M\&S) to Support Intelligence, Adaptation and Autonomy.

Traoré, M. K., Zacharewicz, G., Duboz, R., \& Zeigler, B. (2018). Modeling and simulation framework for value-based healthcare systems. SIMULATION, 0037549718776765.

Zeigler B. P. (1976). Theory of Modeling and Simulation. Wiley Interscience.

Zeigler, B. P., \& Hammonds, P. E. (2007). Modeling and simulation-based data engineering: introducing pragmatics into ontologies for net-centric information exchange. Elsevier.

Zeigler, B. P., \& Sarjoughian, H. S. (1999). Support for hierarchical modular component-based model construction in DEVS/HLA. In Simulation Interoperability Workshop (pp. 14-19).

Zeigler, B. P., \& Sarjoughian, H. S. (2017). Modeling and simulation of systems of systems. In Guide to Modeling and Simulation of Systems of Systems (pp. 3-11). Springer, Cham.

Zeigler, B. P. (1984). Multifacetted modelling and discrete event simulation. Academic Press Professional, Inc.

Zeigler, B. P. (1991). Object-oriented modeling and discrete-event simulation. Advances in Computers, 33, 67-114. 
Zeigler, B. P., Mittal, S., \& Traore, M. K., 2018. MBSE with/out Simulation: State of the Art and Way Forward. Systems 6(4):40,

Zeigler, B. P., Traoré, M. K., Zacharewicz, G., \& Duboz, R. (2019). Value-based Learning Healthcare Systems: Integrative Modeling and Simulation Architecture. London: The Institution of Engineering and Technology.

Zeigler, B.P., Praehofer H., \& Kim T.G. (2000). Theory of Modeling and Simulation Second Edition: integrating discrete event and continuous complex dynamic systems. Academic Press. 